\title{
PROGETTO DI ISTITUZIONE DI DISTRETTI CULTURALI EUROPEI. IL DISTRETTO CULTURALE GOMOSAICO DI GORIZIA
}

\author{
GIULIO M. CHIODI (*)
}

\author{
Nota presentata dal m.e. Silvio Beretta
}

(Adunanza del 14 novembre 2019)

SunTO. - Si presenta la proposta di istituzione di distretti culturali europei con lo scopo di valorizzare congiuntamente il patrimonio culturale e il complesso di attività di territori con particolari caratteristiche degne di riconoscimento e di tutela internazionale. Si illustra l'esempio del distretto in costruzione che fa capo alla città di Gorizia, le cui peculiarità si fondano sulle basi dell'antica e singolarmente originale civiltà di Aquileia, sulle vicissitudini belliche sofferte e sull'unicità di trovarsi area di incontro delle tre principali culture dell'Europa, la latino-mediterranea, la germanica e la slava, quale punto strategico della Mitteleuropa.

$* * *$

ABSTRACT. - It is here presented the hypothesis of establishing European cultural districts. Its purpose is to systematically valorize the cultural heritage of their territories end their people. The proposal is innovative with contribution to economic and civil life. A cultural district with Gorizia as capital is given as significant exemple. Its importance is that it originates from the tradition of ancient Aquileia and that is meeting point between three very important ethnic groups: Latin, German, Slavic. It is a district of symbolic value also for the enhancement of the strategic axis for Europa "MitteleuropaMediterranean”.

(*) Istituto Universitario Suor Orsola Benincasa, Napoli, Italy.

E-mail: giuliomaria.chiodi@uninsubria.it 


\section{IL TEMA}

Ringrazio sentitamente l'Istituto Lombardo di Scienze e Lettere e in particolare il suo Presidente Silvio Beretta per l'invito ad effettuare un intervento presso questa prestigiosa istituzione. Me ne sento profondamente onorato.

Il mio compito è principalmente informativo e riguarda la nascita di un'iniziativa, la cui importanza sta destando l'attenzione ad alti livelli istituzionali e presso diverse autorità costituite, nazionali e internazionali, tra cui dobbiamo annoverare anche quella del Capo dello Stato italiano. Dico subito che si tratta della proposta istitutiva di distretti culturali europei da sottoporre alle sedi competenti dell'Unione Europea, ma io mi limiterò a sottoporre all'uditorio quanto in merito è stato già avviato con successo, grazie soprattutto alla collaborazione con l'ICM (Istituto per gli Incontri Culturali Mitteleuropei di Gorizia). La scelta è doppiamente motivata: da un lato il valore altamente significativo dell'iniziativa, dall'altro si tratta del primo esempio di distretto culturale pilota, la cui configurazione territoriale è stata già ben definita in seguito all'impegnativo e competente lavoro prodigato dall'ICM.

\section{PREMESSA}

Prima di entrare direttamente nell'argomento mi sembra opportuna una breve premessa sul percorso che ha portato a maturazione l'idea. La prima circostanza risale press'a poco al febbraio 2017. Il 9 febbraio è la data stabilita ufficialmente dal governo greco per celebrare la cultura e in particolare la lingua greca, per il grande ruolo svolto nella formazione della nostra civiltà. In quella occasione a Napoli - città, fra l'altro, dalla quale era partito per Atene il suggerimento di istituire tale giornata celebrativa - ero stato invitato dagli organizzatori della celebrazione a parlare della rilevanza dello studio del greco per la filosofia politica e civile moderna. A margine dell'evento ebbi modo di conversare con Lucio Minervini, che già in precedenza avevo avuto modo di conoscere, magistrato con stretti contatti con gli organismi di vertice dell'UNESCO e primo promotore della delibera del parlamento greco sulla giornata della lingua. Mi disse che stava elaborando l'idea di far proclamare dall'UNESCO le lingue e la cultura greca e latina beni immateriali patrimonio dell'umanità. Una tale prospettiva era in perfet- 
ta sintonia con un progetto avviato anni prima da Domenico Conci, filosofo teoretico ed antropologo dell'Università di Siena, purtroppo scomparso prematuramente, alla cui elaborazione avevamo lavorato insieme, e che tra l'altro si proponeva l'allestimento di uno speciale museo informatico presso gli scavi dell'antica Elea nel Cilento. ${ }^{1}$ Elea, patria di Parmenide e terra in cui si può dire che sia nata la filosofia, ossia la scienza greca e quindi, in larghissima misura, culla della cultura e della civiltà dell'intero Occidente; per di più i suoi resti sono situati a poca distanza dagli scavi di Paestum e nell'immediato entroterra sorge il castello di Vatolla, dove Giambattista Vico scrisse, rievocando l'antichità classica, la Scienza nuova. Ripensando a questo antecedente, proposi che l'elaborazione della proposta in questione, ossia di far dichiarare la cultura classica bene immateriale dell'umanità, poteva incominciare a trovare anche un sostegno, per così dire, materiale e coerente in un ben preciso e significativo territorio. E' stato allora che si sostanziò, con i debiti approfondimenti, l'idea di proporre nelle opportune sedi europee l'istituzione di distretti culturali europei, comprendenti aree, le cui peculiarità storiche e qualificatrici presentassero fondati motivi per una loro valorizzazione unitaria.

Si giunse così alla costituzione di un comitato, cooptando anche come esperto di diritto ambientale Giovanni Cordini, che, adite le sedi competenti, ha ottenuto il patrocinio dell'UNESCO con la denominazione di "Comitato Arte e Cultura per l'Economia - Economia per l'Arte e la Cultura" e col compito di promuovere lo studio delle culture e delle lingue latina e greca e l'istituzione di distretti culturali europei. Il suo lavoro è sfociato, fra l'altro, nell'allestimento di un convegno internazionale, tenutosi presso il Museo Archeologico Nazionale di Napoli, nel dicembre del 2018, con la partecipazione di personalità di rilievo e anche con quella, in rappresentanza ufficiale, di alti gradi delle tre religioni monoteiste, tra cui il Rabbino Capo e il Gran Muftì di Bosnia. Nell'occasione sono stati presentati i due obbiettivi del Comitato: pervenire da parte dell'UNESCO alla proclamazione delle lingue e della cultura greca e latina beni immateriali appartenenti al patrimonio dell'uma-

1 Erano già stati presi contatti col sindaco del luogo e si era anche individuato il terreno e preventivati i costi per l'erezione di una struttura, ideata con caratteristiche ispirate alla visione del mondo parmenidea. Cambiamenti della rappresentanza amministrativa e alcune inerzie locali hanno impedito la realizzazione dell'iniziativa. 
nità, ottenere dall'Unione Europea l'istituzione di distretti culturali europei. In merito a questo secondo obbiettivo vennero presentati due progetti di distretto-pilota, nei quali sperimentare l'individuazione dei parametri necessari alla definizione del genere di distretto previsto. L'uno era inerente territori della Campania, Calabria e Sicilia, particolarmente qualificati dalla presenza di continuità dell'antica tradizione classica; il secondo era rappresentato da un'area caratteristica che si estende dalla costa nord-adriatica alla Carinzia centro-meridionale. Questo secondo ipotetico distretto fu già allora provvisoriamente elaborato dall'ICM di Gorizia e presentato da due membri autorevoli del suo direttivo, l'ingegnere Nico Fornasir, friulano vicepresidente dell'Istituto e principale organizzatore del progetto, e il prof. Boris Nemec, sloveno e presidente del Forum za Goriško, che ha sede a Nova Gorica.

\section{SUl DISTRETTO CULTURALE IN GENERALE}

Il mio compito specifico è qui di sottoporre all'attenzione degli illustri presenti le caratteristiche essenziali di questo secondo progetto di distretto.

Innanzitutto è bene tratteggiare un'idea molto generale di che cosa si debba intendere per distretto culturale nel senso adottato dal suddetto comitato.

Ci sono, per esempio, territori che ospitano scavi archeologici o monumenti di particolare rilevanza, concentrazioni di materiale storicoartistico di elevata portata culturale, che l'UNESCO stessa ha riconosciuto patrimonio dell'umanità, oppure che altre istituzioni autorevoli hanno sottoposto a loro tutela; ci sono poi territori che presentano particolari caratteristiche naturali o ambientali, ecologiche come taluni microsiste$\mathrm{mi}$, che godono delle medesime o consimili protezioni; in alcuni luoghi sono già costituiti distretti (ma non formalmente distretti europei), aventi a finalità la valorizzazione di attività produttive molto specifiche, agricole, artigianali o, a vario titolo, economiche. Le tipologie sono svariate, come lo sono altresì gli organisimi istitutivi e di gestione.

L'intento ispiratore del distretto culturale europeo ha il preciso obbiettivo, là dove se ne dà idonea materia, di erigere a sistema, per così dire, le suddette caratteristiche e le diverse componenti che concorrono a qualificare l'identità di particolari ambienti, con lo scopo di tradurle unitariamente in fattori sociali, coinvolgenti anche la consapevolezza 
della popolazione del luogo, favorendo in tal modo un orientamento coerente dei suoi sviluppi civili ed economici. Tra gli effetti attesi, a seconda delle realtà locali, è un accrescimento sia della consapevolezza del proprio patrimonio storico, sia del sentimento di appartenenza nella popolazione che abita nella zona, sia delle relazioni culturali all'interno e con l'esterno. Il distretto, fra l'altro, una volta riconosciuto, sarebbe abilitato ad accedere a fondi di sostegno, previsti dalla politica dell'Unione Europea per la tutela dei beni culturali.

Detto in altri termini, si auspica che il distretto diventi una realtà innovativa, capace di contribuire ad elevare il tono civile del mondo sociale, che lo abita e lo vive. Per esempio, laddove è custodito un patrimonio storico-monumentale importante o dove sussiste un esteso patrimonio di grandissimo pregio, l'opera promozionale del distretto deve far sì che tali presenze non rimangano soltanto oggetto di studio per gli specialisti o oggetto della curiosità, spesso distratta e superficiale, del turista di passaggio, ma che diventino anche un complesso idoneo a stimolare le continuità di appartenenza di una popolazione nonché fonte di attrazione di interessi qualificati. Tutelando e incrementando le eredità locali ed ospitando iniziative di richiamo coerenti con la sua natura ambientale, il distretto salvaguarda un'identità collettiva, mettendone a frutto tutte le potenzialità e nel contempo contribuisce ad integrarle nella vita collettiva, elevandone il tono civile. Il nostro paese, per esempio, è particolarmente ricco di tali disponibilità.

Ovviamente, per pervenire ad un riconoscimento ufficiale di questa nuova entità è necessario che si stabiliscano i requisiti per determinare i parametri indicativi delle sue specificità. Ma questi sono elencabili, naturalmente, solo caso per caso, presentando ogni ipotetico distretto situazioni completamente diverse da quelle di altri. Inoltre, dobbiamo aggiungere che le indicazioni nel merito possono provenire soltanto dal basso, ossia dalle concrete realtà e dalle istituzioni operanti in loco. Perciò ora non posso che enunciare il principio ispirativo più generale della costruzione della tipologia di distretto di cui ci stiamo occupando e interpretare il ruolo che la sua costituzione potrebbe comportare.

Parliamo di distretto culturale europeo e perciò esso è definito dai due aggettivi. Già esistono, infatti, vari tipi di distretti: economici, industriali, legati a parchi regionali o nazionali, o a contesti ambientali, ecosistemici o di particolare fisionomia paesaggistica o anche produttivosettoriali. Ma nessuno di questi è distretto culturale, nel senso storicosociale del termine, né ha ottenuto riconoscimento europeo, come è 
invece quello che ci si è proposto come obbiettivo. Culturale nel nostro caso è aggettivo adottato nel suo duplice significato, cioè sia nell'espressione che designa valori di alta formazione e qualificazione, sia in quello che allude a modalità, attitudini e consuetudini di vita comuni che caratterizzano una collettività. Cultura, dunque, in senso verticale, ossia gerarchico, e cultura in senso orizzontale, ossia comunemente partecipata. Potremmo anche ricorrere contemporaneamente, forse con qualche enfasi, ai due concetti espressi in tedesco coi termini di Zivilisation e Kultur. Quanto all'aggettivo "europeo" qui si spiega appunto con la proposta di riconoscimento del distretto da parte dell'Unione Europea, in quanto realtà dotata di valenze di rilevanza comune per l'intera Unione.

Mi soffermerò, come detto, a fare cenno del progetto di distretto che ha preso le mosse dalle specificità che riveste la città di Gorizia. La definizione di precisi parametri qualificativi di questo contesto territoriale è tuttora in corso di elaborazione, perciò non sono in grado di fornirne elenco e caratteri, ma soltanto i principî orientatori.

\section{Sul DisTRETTO GoMosAico}

Gorizia è innanzitutto un'eccezionale città simbolo, che tutti sanno ancora oggi divisa da un confine di stato, che separa territorio italiano da territorio sloveno. Quel confine, anche se tutt'oggi sussiste a tutti gli effetti, ha enormemente attenuato le sue rigidità, consentendo libero transito ai cittadini. Tracciato materialmente a squadra e righello dopo la fine della seconda guerra mondiale, di questa ha continuato la follia, non limitandosi soltanto a troncare in due parti la città, sottoponendola alla giurisdizione di due diversi stati, ma arrivando addirittura a separare ambienti di una medesima abitazione, sì che qualcuno, per esempio, era costretto in casa propria ad avere la camera da letto nell'allora Jugoslavia e la cucina o la sala da pranzo in Italia. Si è giunti al punto, piuttosto allucinante, di tormentare perfino i defunti, violentando un cimitero in modo da tagliare in due altresì talune tombe. Ancora oggi i segni di questa assoluta follia sono stati lasciati visibili per poterli mostrare a visitatori, quanto meno sbigottiti di quanto cade sotto il loro sguardo incredulo.

$\mathrm{Ma}$ Gorizia è città simbolo non solo per aver patito questi assurdi oltraggi - come del resto è accaduto soprattutto con città tedesche, pur con le differenze del caso e con relativamente analoghe motivazioni punitive - ma perché il suo più recente stravolgimento è per molti versi 
una prosecuzione di quanto sofferto durante la tragedia della prima guerra mondiale, di cui la seconda può considerarsi un quasi naturale prosieguo. In quei terribili frangenti Gorizia e i suoi immediati dintorni sono stati, come è noto, il teatro di una delle più imponenti e sanguinose guerre, anche fratricide, con centinaia di migliaia di morti. La città si trovò ripetutamente sotto il fuoco dell'uno e dell'altro fronte, alternativamente occupata, con le distruzioni e gli eccidi che si possono immaginare, ora per opera degli italiani e ora per opera degli austro-ungarici; ma non è solo questo, se si aggiunge che gran parte delle sue famiglie, come accadde del resto anche altrove, ebbe al suo interno chi dovette indossare una divisa militare e chi la divisa nemica di questa.

Vicende di tale natura, che abbiamo detto fare di Gorizia una speciale città simbolo, sono espressione di una storia che coinvolge l'intera Europa e che trova nella città la sede di memorie fortemente rappresentative per tutti. Ma la natura simbolica del luogo possiede anche altre caratteristiche, che giustificano di averla identificata come riferimento altamente significativo per essere scelta quale sede principale di un distretto culturale europeo.

Le importanti vicende che la città ha attraversato nel secolo scorso si innestano, infatti, in un territorio e in una storia che le ha precedute e per di più con salde e robuste radici, la cui linfa è stata deviata $o$ ne è stato interrotto il flusso soprattutto dal sopravvento prepotente dei nazionalismi. Parliamo in specie di un territorio e di una storia che uniscono tradizioni e caratteristiche culturali di popolazioni diverse, che possiamo far risalire a una comune matrice unificante e che dobbiamo riconoscere furono, nelle loro linee essenziali, salvaguardate fin che durò l'impero asburgico. Si tratta della civiltà sorta, sviluppata e poi diffusa sotto l'egida di Aquileia e del suo Patriarcato.

Non è certo questo nostro incontro l'occasione per illustrarne la storia, ma tutti dobbiamo ricordare che Aquileia, città di fondazione romana e importantissimo porto all'estremo nord del Mare Adriatico, fatta capitale della Regio $X$, è stata fin dai suoi inizi un punto nevralgico di convergenze, dal quale si dipartivano le vie terrestri che più direttamente conducevano all'arco delle Alpi e che da quest'ultimo, attraversavando le province della Rezia, del Norico e della Pannonia, raggiungevano il limes danubiano dell'impero. Aquileia e il suo porto divennero il principale punto di congiungimento tra le aree centro-continentali, le zone adriatiche e ioniche, quelle greche, del Medio Oriente ed egizie. La grande città, oggi ridotta ad un comune di poco più di tremila abi- 
tanti, divenne nei primi secoli d. C. un vivissimo centro di diverse culture e infine fu ben presto un decisivo punto di riferimento del primo espandersi del cristianesimo. Si può dire che soprattutto da qui partì l'evangelizzazione di buona parte del nord, e di tutto il nordest ed est del continente. Non è superfluo precisare che l'acquisizione aquileiese della nuova religione non fu di origine petrina, bensì alessandrina, favorita dal particolare asse marinaro stabilitosi con Alessandria d'Egitto: la tradizione vuole infatti che la Chiesa di Aquileia sia sorta direttamente ad opera dell'evangelizzazione di San Marco. Il Patriarcato, in realtà, sorse con forti impronte miste di stampo romano e orientale, che influenzarono il suo tipo di teologia, conferendogli una interessante originalità, e nel pieno della sua massima espansione raggiunse una vastità straordinaria, che lo portò a incorporare le coste nordadriatiche, il Friuli e a comprendere al nord gran parte della Carinzia, mentre a Est arrivò fino al Danubio e a ovest fino a Como e al suo circondario. Diciamo che si è trattato di una delle maggiori circoscrizioni d'Europa, che sotto il profilo solo ecclesiastico si protrasse fino al 1751, data della sua cessazione e dell'istituzione delle tre diocesi, che ne hanno raccolto l'ultima residua eredità: Gorizia, Udine e Klagenfurt. Da sottolineare, inoltre, che il patriarcato non fu solamente, già dal IV secolo d. C., il principale centro di irradiazione della religione cristiana nel continente, ma in seguito all'ottenimento di un'investitura imperiale (ci riferiamo al Sacro Romano Impero), che perdurò fino al 1420, potette esercitare anche pieni poteri civili, che gestì con criteri molto interessanti soprattutto la distinzione tra funzioni religiose e funzioni civili. Fra l'altro, merita di essere menzionata l'istituzione di un parlamento (lo si può considerare tra i più antichi), con partecipazione popolare fondata su base territoriale con caratteri pressoché federali e, come ben documentato, tipico anche per la sua pratica multilingue.

Bastano, ritengo, le poche indicazioni ora richiamate alla nostra memoria su antefatti più o meno remoti per giustificare la scelta di Gorizia come sede principale del distretto, essendo altresì la più vicina erede della tradizione aquileiese. Mi soffermo, come preannunciato, sul progetto costitutivo di distretto culturale europeo che la riguarda.

Il fattore caratterizzante principale del distretto goriziano, e assolutamente unico nel suo genere, è oggi quello di essere il punto di incontro delle tre principali etnìe, per così menzionarle, componenti la compagine europea: la germanica, la slava, la latina, ma con vari innesti minori. Sotto il profilo strettamente geografico, le estreme propaggini 
dei territori occupati da queste popolazioni si congiungono press'a poco a un terzo dalla frontiera nord che delimita il previsto distretto. E'infatti un vero e proprio simbolo di natura geografica di questa unione la famosa Dreiländerecke, formata da picchi rocciosi e situata alla congiunzione delle due catene delle Alpi Giulie e delle Caravanche, precisamente laddove Friuli, Slovenia e Carinzia si saldano vicendevolmente.

La caratteristica di raggruppare le frange estreme di quelle tre grandi popolazioni fa idealmente del distretto, più di ogni altro, un vero cuore rappresentativo di tutta l'Europa. Si tratta di tre componenti etniche che hanno dato vita anche ad altrettante importanti nazionalità e che nel passato si sono scontrate le une contro le altre, mentre ora più che mai, nella riscoperta della loro comune identità, possono essere vantaggiosamente determinanti per se stesse e per l'intera Europa: conseguire una stretta collaborazione, data anche l'indiscutibile comunanza dei destini nell'affrontare il difficile presente, e ancor più le pesanti incognite serbate dal futuro, significa costruire un punto di forza esemplare sotto tutti i punti di vista. In realtà, oltre alle tre suddette etnie, il medesimo territorio ha anche ospitato, tempi addietro, l'estrema appendice orientale della cosiddetta mezzaluna celtica, di cui non mancano reperti archeologici. A parte poi alcune entità minori, sono ancora oggi vive le tracce della forte presenza ebraica, tragicamente annientata da una forma di violenta follia, aggiuntiva a quella già sconvolgente delle guerre ivi combattute. Comunque, è soprattutto il congiungersi delle estreme ali delle tre principali etnie europee che rendono straordinariamente e in maniera indiscutibile unico e grandemente rappresentativo il luogo che abbiamo preso in considerazione.

Posto che vi si incontrano gli insediamenti tedeschi, slavi e italolatini, non sarebbe del tutto rispondente a realtà considerare che l'area distrettuale di cui parliamo sia da definire semplicemente come punto di convergenza di tre diverse culture. Mi sembra molto più appropriato, invece, alle effettive condizioni del contesto in questione la precisazione avanzata da Hans Kitzmüller: non si deve parlare di più culture all'interno di questa area, bensì di una sola e indivisibile cultura strutturata sul plurilinguismo. ${ }^{2} \mathrm{E}$ non è affatto arbitrario sostenere, come più volte tempi addietro è stato scritto di Gorizia, che era una città tedesca

2 Cfr. Hans Kitzmüller, Una chiosa per conoscere storia e territorio. Paesaggi della memoria e dell'oblio, in "Iniziativa isontina", n. 79 (aprile 2019), p. 12 sgg. 
nella quale chiunque, anche di umili origini, sapeva esprimersi correntemente in tre lingue (ossia in tedesco, in schiavone o slavone (sloveno) $\mathrm{e}$ in furlano o italiano). E questo è certamente un altro requisito che contribuisce a conferire un valore simbolico a questa città, fra l'altro tutt'altro che priva, per altre ragioni, di un suo sottile fascino.

Ma Gorizia per molti versi è anche un'erede, come dicevamo, privilegiata della tradizione di Aquileia, essendo diventata, nel quadro espansivo del "Patriarcato-Principato" aquileiese, la capitale di un ducato e poi contea, che estendeva la sua giurisdizione fino a comprendere un buon tratto del territorio carinziano. Per portare un esempio di presenza simbolica quanto mai efficace in tal senso, mi limiterò a ricordare un massiccio cimelio medievale, risalente proprio al periodo di auge goriziana; è ubicato nella zona di Zollfeld a meno di una decina di chilometri da Klagenfurt. Si tratta di un rustico doppio trono con unico schienale, costruito con poche grosse pietre scalpellate, materiale di riporto prelevato dalle rovine della vicinissima città romana di Virunum, che fu capitale di quella zona. Un lato di quel trono era riservato alla massima autorità del ducato di Carinzia; sull'altro lato sedeva la massima autorità della contea di Gorizia, che estendeva la sua giurisdizione sui territori occupati soprattutto da popolazione di lingua slovena. Ivi veniva celebrata congiuntamente l'alta giurisdizione di entrambe. Ancor oggi il monumento, chiamato comunemente lo Herzogstubl, ha conservato uno speciale valore rappresentativo ed è spessissimo visitato da scolaresche appositamente accompagnate, provenienti per lo più da diversi luoghi della Slovenia.

Per non tergiversare in particolari sparsi, cercherò di riassumere le linee generali ispirative del progettato distretto culturale, che gli estensori locali, guidati dall'ICM, hanno definito "GoMosaico", e redatto in tre lingue (italiano, sloveno e tedesco), anche con la partecipazione del comitato italo-sloveno per la candidatura di Nova Goriza, col sostegno della Gorizia italiana, a capitale europea della cultura per l'anno 2025.

Innanzitutto, sotto il profilo geografico, l'estensione del distretto è stata a grandi linee precisamente definita, ed è molto vicina a coprire un territorio più o meno accentrato sull'antica contea goriziana. I suoi confini delimitativi devono essere naturalmente interpretati in maniera alquanto elastica ed esenti da vere e proprie restrizioni. Si tratta di un'area culturalmente e non amministrativamente intesa, con tutte le permeabilità del caso; diciamo che è stata individuata un'area propulsiva. Da nord a sud, approssimativamente, si va dalla zone carinziane del Gaital e di Villaco a Klagenfurt e a tutto il suo circondario fino più o 
meno a Völkermarkt, coprendo la Carinzia centro-meridionale; superate poi le alture delle Caravanche, si scende, all'incirca tra i dintorni del Monte Santo di Lussari a ovest e la località di Jenice ad est, lungo tutta la valle dell'Isonzo in Slovenia, coprendo anche verso occidente una larga fascia del Friuli fino a raggiungere il mare con Grado, Monfalcone, Duino-Aurisina, mentre in direzione sud-est è compreso un ampio tratto della Slovenia fino a Idra e a Aidovš ina (denominata, quest'ultima dai romani Castra ad flumen frigidum).

Veniamo ai concetti chiave qualificativi del distretto, che cercherò di sintetizzare come segue.

Prima di tutto è da sottolineare l'incrocio storico di popolazioni di diversa origine che, senza perdere la propria identità hanno saputo costruire una nuova e speciale forma di civiltà, che porta in sé le nitide tracce di origini greche, romano-latine, bizantine, germaniche, slave, illiriche, convergenti in un'unità multiarticolata, esprimente l'essenza stessa della cultura e dello spirito mitteleuropei. Queste qualità, tuttora vitali nonostante le vicissitudini trascorse, sono una premessa per armonizzare un dialogo e un costante interscambio tra popolazioni che si autointerpretano in quattro lingue (italiano, sloveno, tedesco e friulano), unite da un'unica storia e un unico costume, nonché da memorie comuni e medesime condizioni economiche locali. Il distretto recepisce e deve contribuire ad ottimizzare queste qualità.

Il distretto può essere concepito come una rete di istituzioni pubbliche e private, associazioni, organizzazioni o imprese, collegati tra loro dall'interrelata gestione di un patrimonio comune da tutelare e da potenziare in funzione civile, con attenzione altresì alle esigenze economiche. Nel patrimonio di GoMosaico sono riconoscibili, oltre a un significativo numero di siti archeologici, anche preziose opere architettoniche, castelli e dimore storiche, che affiancano importanti creazioni artistiche e raccolte di pregio e che documentano precisi valori e continuità culturali. Si riscontrano, inoltre, peculiarità di natura paesaggistica (talune anche a seguito di intervento umano, e molte rese oggetto, accanto a personaggi leggendari, di famosi racconti popolari) o di carattere agricolo, o gastronomico ed ecologico in generale, che costituiscono fonte anche di attrazione culturale ed economica di non comune rilevanza. La realizzazione di una rete di collegamento di tutte queste realtà e potenzialità, distribuite in varie località del territorio e attualmente soggette a una giurisdizione suddivisa tra tre diversi stati nazionali (Austria, Italia, Slovenia) porrebbe in atto fortissimi incentivi, tanto 
alla sperimentazione di nuove forme di collaborazione e di interazione quanto all'incremento di innovazioni e di interventi creativi, congiungenti il passato col presente e contribuendo alla costruzione di un futuro non soltanto da attendere fatalmente e da essere costretti a subire.

Negli auspici è determinante la valorizzazione di esperienze che attingono direttamente alle energie sorte o cresciute sul posto, a garanzia di modalità di interscambio e di integrazione che non distruggono genuinità e tradizioni, dotate ancora oggi di robusta consistenza e socialmente bene qualificanti, ma minacciate dalle dilaganti dinamiche globalizzanti. Ne deriverebbe un consistente incentivo alle risorse e un sicuro apporto nell'indirizzare le strategie territoriali in merito alle loro tipologie produttive, industriali, manifatturiere, agricole e variamente gastronomiche, nonché al sostegno e alla migliore qualificazione del turismo, compreso il potenziamento di tutte le necessarie infrastrutture.

Un'aspettativa particolarmente indicativa è rivolta alla formazione dei giovani. Si tratta di stimolare le istituzioni competenti a renderli fin dall'infanzia consapevoli di un'appartenenza culturale importante, la cui cura e fortuna sarà ben presto affidata proprio a loro. Fare conoscere alle nuove generazioni la storia, l'arte, i monumenti, le caratteristiche fondamentali e le peculiarità di portata più elevata, ma anche il tipo di economia caratterizzante e le più importanti tradizioni delle loro terre è un compito che il distretto contribuirebbe decisamente ad assolvere in collaborazione con le istituzioni interessate e, in primo luogo, ovviamente con le scuole e con l'università.

Rientra nella strategia distrettuale il rafforzamento degli aspetti identitari e nel contempo lo stimolo al confronto interlinguistico, intrapreso dal vivo e dal vissuto quotidiano fin dai primi anni di vita. E' un impegno formativo che favorisce l'apertura mentale e sviluppa utilmente le attitudini critiche e autocritiche. Mi permetto di citare in tema due esempi, che trovo illuminanti.

Il primo è aneddotico. Ervino Pocar, grande traduttore e intellettuale di altissimo profilo, frequentò da studente l'allora asburgico Staatsgymnasium, il prestigioso liceo classico di Gorizia; egli ha più volte ricordato l'arricchimento intellettivo dei ragazzi, tratto da un corso di lingua francese, tenuto a studenti italiani in lingua tedesca da un insegnante di madrelingua slovena. Come secondo esempio porto quello del logico e linguista Friedrich Mauthner, importantissimo studioso, da molti ritenuto il principale precursore della filosofia analitica contemporanea, che esercitò altresì molta influenza sul pensiero di Ludwig Wittgenstein. 
Mauthner lasciò scritto che fin da bambino si sentì molto incuriosito dal fatto che un medesimo oggetto o un medesimo pensiero si potesse esprimere con parole profondamente diverse. Cresciuto in un paese della Boemia, in famiglia parlavano in tedesco, ma a scuola e con gli amici di gioco o con frequentatori di casa si parlava abitualmente in ceco. Ritenne decisiva questa esperienza della sua infanzia per gli studi filosofici e non solo di filosofia dele linguaggio, a cui poi dedicò la sua attività.

Il plurilinguismo di base e di uso corrente è considerato fondamentale per conseguire gli scopi che animano il distretto e i suoi promotori sono unanimemente convinti che il distretto stesso debba diventare uno strumento impegnato nella diffusione della pratica delle sue quattro lingue (tedesca, slovena, italiana e friulana). Si potrebbero accumulare citazioni a non finire su queste tematiche, ma qui devo soltanto sottolineare la rilevanza presentata da un plurilinguismo particolarmente connaturato all'ambiente, la cui valorizzazione non si riduce solo alla conservazione di un fattore tradizionalistico, ma è vista come elemento propulsivo di interrelazione e di apertura di orizzonti intellettuali. La lingua non è solo una somma di parole e le parole non servono solo da etichette a cose e pensieri.

Da quanto detto si evince che il distretto culturale, preso nel suo complesso, deve provocare un concorso organizzato di energie e di risorse, in armonia con le amministrazioni pubbliche che governano $\mathrm{i}$ paesi interessati, chiamando in causa istituzioni del luogo, associazioni private, strutture di economia locale, enti dediti alla formazione e ad attività culturali, istituti di ricerca o enti a vario titolo promozionali, musei, teatri, biblioteche, mostre, allestimenti di fiere, manifestazioni celebrative e altri eventi significativi, avviando e incrementando in tal modo una intensa rete di iniziative qualificanti l'intero territorio. Va da sé che i risultati darebbero il loro significativo apporto anche a tutte le altre realtà esterne che verrebbero in contatto col distretto. Sono indiscutibili i vantaggi che ne trarrebbero svariati ambiti attivi anche in altre zone geografiche, in primo luogo e non unicamente europee.

\section{OSSERVAZIONI CONCLUSIVE}

Prima di concludere ritengo che sia opportuno aggiungere una riflessione sul significato che rivestirebbe la realizzazione di un distretto culturale europeo, secondo le linee che sopra ho cercato di sintetizzare. 
Il distretto culturale, in quanto tale, prescinde da qualsiasi strategia politica in senso stretto, non ha assolutamente nessuna coloritura di parte, e per sua natura nasce per nulla incline ad abbracciarne l'una piuttosto che l'altra, ma certamente può provocare indirettamente effetti anche in questa direzione. Ed è auspicabile che lo faccia, a mio parere, ma soltanto nell'ambito di una visione generale che l'Unione Europea dovrebbe avere di se stessa, ma che tuttora mostra di non possedere. Che l'Unione stia attraversando momenti di crisi e di confusione identitaria, versando in difficoltà nel riconoscersi e nell'adottare precise prospettive, non sfugge a nessuno. Uno dei motivi fondamentali dei suoi non leggeri disagi, e probabilmente il principale, è che l'Unione ha sempre tenuto in scarsissima considerazione la reale natura delle popolazioni su cui estende il suo governo e delle loro tradizioni, confermandosi incapace di incontrare e interpretare i loro effettivi sistemi di vita e di relazione. L'Unione troppo spesso interviene con decisioni che assomigliano a terapie applicate ad organismi, dei quali ignora completamente la fisiologia. In ciò è certamente troppo condizionata dall'eccessivo statocentrismo delle sue componenti. Gli europei, detto altrimenti, non sono considerati nelle forme culturali e nelle continuità storiche che li esprimono. Mi viene sempre in mente, in proposito, la frase attribuita a Massimo D'Azeglio: "Abbiamo fatto l'Italia, ora facciamo gli italiani". Ma chi avrebbe fatto l'Italia, se non c'erano ancora gli italiani? Ora sembra che si dica: "Abbiamo fatto l'Europa, ora facciamo gli europei". E chi avrebbe fatto l'Europa, posto che non ci siano ancora gli europei?

I distretti culturali europei non possono e non devono essere pensati e programmati dall'alto, ma vanno concepiti come una diretta manifestazione di quanto già opera dal basso, cioè di quanto vive e si sviluppa spontaneamente sul territorio. Perciò i distretti culturali diventano indicatori profondamente realistici della ricchezza materiale e ideale, creativa e potenziale, colta nelle varietà e nelle multiformità veramente partecipate e vissute autenticamente dalle popolazioni, che è caratterizzante del grande quadro generale europeo. Per questo aspetto l'istituzione di distretti culturali europei offre a tutta l'Europa, presa nel suo complesso, un'occasione di ripensamento e di consolidamento sulle sue reali radici, che indubbiamente giova ad alimentare i sentimenti di appartenenza, di cui ora pesantemente difetta, con conseguenze che inficiano gli indispensabili equilibri interni.

Nel caso specifico del distretto sopra descritto si tocca proprio un punctum dolens. Questo distretto si identifica simbolicamente e 
altresì di fatto con una particolare centralità mitteleuropea; esso è infatti piena espressione di una società che senza esitazione possiamo definire mitteleuropea. E qui dobbiamo precisare. Ci sono stati in passato coloro che nella parola Mitteleuropa leggevano una sorta di egemonia germanocentrica. Non è affatto questa la prospettiva che qui fa da guida. Per mitteleuropeo si deve intendere quel territorio idealmente, direi meglio "spiritualmente" se non venissi frainteso, centrale nella compagine complessiva europea, che sarebbe errato intendere come realtà perimetrata da un confine che lo delimiti e lo circoscriva. Mitteleuropa significa un territorio multietnico e aperto, fatto di confini soltanto interni, simili a ponti intercollegati e non a muri divisorî. A rigore non si tratta nemmeno di confini geografici, anche se in parte lo sono, ma prettamente costumali, tipici di un mondo plurilingue, che possono riferirsi semplicemente anche ad affetti e sentimenti personali e soprattutto ad appartenenze famigliari, da generazioni insediate in quei luoghi. I territori che comprendono l'area distrettuale che abbiamo descritto riflettono tradizionalmente proprio queste caratteristiche, non ancora del tutto cancellate né dai nazionalismi, né dai processi della globalizzazione.

La Mitteleuropa, così concepita come compagine culturale, è altamente rappresentativa e strategica per l'intera Europa. Va preso atto, in proposito, di due precise motivazioni. La prima è motivazione di principio: le aree di cui stiamo parlando segnano l'incontro, come dicevamo, delle principali nazionalità storiche che convergono a costituire l'Europa. La seconda è motivazione pratica: si deve ammettere che la politica dell'Unione Europea, soggiogata dalle grandi strategie mondiali, che tendono a polarizzarsi tra una centralità nordamericana e una centralità asiatica (Cina, India), ignorano completamente o forse anche accantonano deliberatamente il ruolo catalizzatore dell'area mitteleuropea, assecondando con ciò anche indesiderate involuzioni nazionalistiche proprio nei paesi mitteleuropei. Ne è compromesso il ruolo europeo stesso di mediazione tra oriente ed occidente, garantito da una posizione di relativa equidistanza (alla quale sta provando di arrivare la Russia). Ora i paesi mitteleuropei assomigliano a un punto di unione di più periferie, che però dispongono di tutte le condizioni e di tutte le potenzialità per sviluppare particolari dimensioni di maggior centralità, il cui conseguimento si tradurrebbe in una proficua convergenza di energie di portata squisitamente europea. Sono predisposti ad essere robusti centri di gravitazione e di trazione. 
Se prendiamo in considerazione la straordinaria rilevanza dell'intera area mitteleuropea, infatti, non ci si arresterebbe soltanto alle sue proprietà interne. Si pensi alla collocazione geopolitica dei suoi territori. Qui gli assi longitudinali e latitudinali di Europa si incontrano proprio nelle zone sulle quali più di altre si scaricano le principali tensioni tra il nord e il sud del continente, ma soprattutto quelle molto più perturbanti e spesso minacciose tra il suo est e il suo ovest. Proprio lì si incontrano in territorio europeo l'oriente e l'occidente con i contrasti più caldi e problematici. E' fuor di dubbio che si tratta delle aree sulle quali maggiormente si ripercuotono le instabilità dei rapporti inter- e transeuropei. Sotto il profilo degli assi geopolitici dell'Unione Europea l'area mitteleuropea occupa in assoluto il punto centrale, cosicché la sua periferizzazione si ripercuote inevitabilmente sul resto dell'Europa. E questa non è certo l'ultima delle cause del graduale declino del peso decisionale dell'Unione nel quadro complessivo della politica internazionale. Ma rimarrebbe troppo incompleto il quadro sulla posizione strategica dell'area mitteleuropea senza fare un dovuto cenno anche all'area mediterranea. ${ }^{3}$

L'area mediterranea, il sud dell'Europa, versa infatti in condizioni che, fatte le debite specificità e peculiarità locali, presentano singolari analogie con quelle della Mitteleuropa.

Prima di tutto il Mare Mediterraneo è depositario delle più antiche culture che hanno dato origine alle forme di civiltà più evolute di tutta Europa. Di gran lunga prioritari sono gli apporti delle culture greca e romana, che unitamente hanno generato gli strumenti della civilizzazione dell'intero continente. La prima, soprattutto per aver aperto le vie del sapere; la seconda, che della prima ha assimilato le conoscenze, soprattutto per averle rinnovate e diffuse, anche elaborando in una originale e potente chiave universalistica i principî del diritto e l'organizzazione istituzionale della vita associata tra popoli di costu-

3 Mi permetto di segnalare che fin dagli inizi degli anni ' 90 ho incominciato a dedicare attenzione all'importanza, completamente trascurata, di una politica culturale coinvolgente la complementarità storica e strategica dei territori mitteleuropei e mediterranei, attraverso i cui paesi è scorso il flusso fondativo e vitale della civiltà europea. Cfr. Giulio M. Chiodi, L'asse medio d'Europa, in "1989. Rivista di Diritto Pubblico e Scienze Politiche”, a. III, n. 3, 1993, pp. 259 - 543, ripresi nel volume s.a., Europa. Universalità e pluralismo delle culture, Giappichelli, Torino, 2002. Cfr. anche s.a. L'Europa tra Oriente ed Occidente e il suo asse medio, in "Europea", n. 1, 2016, pp. 11-38. 
manze diverse. Non sono poi da trascurare gli apporti di altre culture, diciamo di origine extra-europea, fiorite lungo le coste mediterranee (per esempio ebraica e musulmana), europeizzate per lo più tramite il filtro e l'assimilazione operati dalle due precedenti.

Prima analogia che dobbiamo riconoscere tra Mitteleuropa e Mediterraneo, quindi, è che ai paesi mediterranei dobbiamo riconoscere importantissime ascendenze, riconducibili ai popoli fondatori della civiltà europea, così come si è sottolineato per quelli mitteleuropei.

La seconda analogia riguarda proprio le condizioni strategiche delle aree mediterranee, continuamente esposte a conflitti, crisi economiche, ribaltamenti istituzionali, emigrazioni, tutti eventi provocatori di squilibri internazionali, che prima di tutto investono gravemente le relazioni intra- ed axtraeuropee. In maniera forse ancora più problematica e preoccupante che nell'ambito mitteleuropeo, qui si ripetono le tensioni nord-sud e ed est-ovest, che si riversano confusamente sulla vita del nostro continente.

La terza analogia si ravvisa nella relativa periferizzazione riservata ai paesi euro-mediterranei dalla visione strategica seguita attualmente dalle potenze dominanti e sostanzialmente seguita dalla politica generale dell'Unione Europea. E' il medesimo atteggiamento che abbiamo riscontrato nei confronti dei paesi mitteleuropei.

Le analogie ora messe in evidenza ci inducono ad avanzare una considerazione della massima rilevanza. E' sufficiente una semplice constatazione geostrategica per rendersi conto della stretta complementarità intercorrente tra le due suddette grandi aree, che sono espressione della più radicale europeità. Collegate tra loro dal corridoio marittimo del Mare Adriatico, costituiscono, per così dire, i due poli di una compagine pluriregionale fortemente interdipendente. L'area mediterranea svolge la funzione di confine e sbocco marittimo meridionale del nostro continente, mentre l'area mitteleuropea ne rappresenta il naturale retroterra continentale più esteso e al tempo stesso più centrale. Gli assi mediani nord-sud ed est-ovest convergono su tale polarità, poiché il principale asse nord-sud d'Europa attraversa la Mitteleuropa e tutta la linea di tensione oriente-occidente che investe la compagine europea ha sede lungo i paesi di entrambi i poli. Se l'Europa difetta, come è indubitabile che stia accadendo, di un proprio baricentro effettivamente rappresentativo della sua realtà culturale (uso l'aggettivo in senso onnicomprensivo), essa lo può trovare proprio e 
soltanto nei territori gravitanti sulla linea che unisce il Mediterraneo alla Mitteleuropa. ${ }^{4}$

Termino questa riflessione integrativa con due affermazioni, che ritengo molto fondate.

L'una guarda all'Europa nella sua unità. Per mantenere un ruolo non subalterno e responsabile del proprio futuro nel quadro della politica mondiale, essa deve saper recuperare le radici della propria cultura, che sono alla base della sua civiltà e delle potenzialità delle sue risorse, rinnovandone le energie per se stessa e per il resto del mondo. Questo orizzonte le è dischiuso dal rafforzamento delle linee di trazione culturale che qui abbiamo indicato; per riscattarsi dalla periferizzazione,

4 L'importanza dei centri di gravitazione e delle linee di trazione costituiti dal complesso Mitteleuropa-Mediterraneo sembrano essere stati ben compresi dagli strateghi della politica estera e commerciale della Cina. La Cina, impossessatasi del Pireo, il porto di Atene, base importante per il Mediterraneo europeo orientale, si sta impegnando per fare altrettanto col porto di Trieste, controllando in tal modo i movimenti commerciali su tutto il corridoio adriatico. Nel frattempo ha posto lo sguardo anche sulla vecchia linea ferroviaria della Transalpina, che i confini imposti dai trattati di pace, già dopo la prima guerra mondiale, hanno completamente emarginato, sconvolgendo l'originario sistema di vie ferrate, programmato dall'impero austro-ungarico. Da notare che si tratta della linea più breve in assoluto che congiunge il Mediterraneo al centro Europa. Ma parlavamo anche della svalutazione che gli orientamenti praticati dall'Unione mostrano di avere nei confronti delle sue più solide radici culturali, di cui le più importanti risalgono senza dubbio al mondo classico. Chiamata ora in causa la Cina, è certamente straordinario constatare come i suoi interessi tipicamente commerciali siano accompagnati anche da una particolare attenzione per le remote radici della nostra cultura. Cito, a titolo di esempio, due diversi ma evidentemente interconnessi fatti. E' noto che in Cina, anche in vista di sue riforme ordinamentali, si stia studiando e traducendo i testi del diritto romano. Ma attraverso questa ricezione - come in una conversazione privata mi ha confermato Francesco Casavola, che nel merito ha presieduto una commissione speciale - si vuole anche apprendere quali aspetti normativi abbiano a loro tempo favorito l'espansione civile di un grande impero. Un'attenzione, ancorché prevedibilmente strumentale, molto lodevole. Il secondo caso riguarda una mia esperienza personale, che mi ha profondamente colpito. Invitato a tenere una lezione presso il Vivarium Novum di Frascati, meritevolissima istituzione dedita alla valorizzazione della cultura classica e dei suoi contributi umanistici, mi sono trovato accolto da una sessantina di cinesi in visita a Roma, all'apparenza tra i dieci o dodici e i quarantacinque anni, che con mia spiazzante meraviglia hanno preso a conversare non nella lingua di Trump, come chiunque si sarebbe aspettato, bensì in quella di Cicerone. Ascoltare cinesi parlare in latino deve portarci tutti a riflettere profondamente su noi stessi. Mostrando di imparare da noi, ci stanno insegnando a casa nostra quello che noi dovremmo essere e fare; ed è piuttosto desolante. 
verso la quale viene sempre più sospinta, l'Europa dovrebbe assolutamente porre un'attenzione operativa e una speciale cura agli assi strategici ora delineati. Per agire a tal fine occorre ridistribuire e meglio valorizzare i baricentri culturali che fungono da nuclei traenti dell'intero sistema e non c'è dubbio che tra questi baricentri un particolare ruolo propulsivo va riconosciuto all'asse medio strategico MitteleuropaMediterraneo, imponendo la necessità di aiutare i paesi che lo occupano a sottrarsi dall'inerzia o da possibili regressioni, sia particolaristiche che globalizzanti.

Una grande responsabilità in merito al potenziamento di quest'asse investe soprattutto lo stato italiano che, attestato alla Mitteleuropa nell'arco alpino e lungo il nord dell'Adriatico, ed estendendosi poi verso sud ad occupare la posizione assolutamente centrale nel Mediterraneo, funge da naturale ponte geo-strategico, ma anche geostorico, di congiungimento delle due grandi aree. La totale noncuranza della sua posizione e dei suoi risvolti strategici in merito, nonché la sua debolezza e sottovalutazione nel quadro europeo e internazionale, si evince dalle scelte politiche da esso compiute. Basti dire che non accade mai che la questione qui posta, nonostante la sua enorme portata, venga minimamente sfiorata, né dai governi, né dal dibattito intercorrente tra le forze e le rappresentanze politiche del paese.

Concludo il mio intervento. Ovviamente la proposta istitutiva del distretto culturale GoMosaico prescinde completamente dai riferimenti strategici che ora ho tratteggiato ed è assolutamente e volutamente estranea a qualsiasi indirizzo ideologico e politico, quale ne sia il colore; essa cade tuttavia in un ambito che sollecita prospettive di sfondo che da parte mia ho cercato di indicare e che considero essenziale per avviare un futuro per i nostri paesi non soltanto eterodiretto da interessi estranei o, peggio, oppressivi, sfruttatori e strumentalizzanti.

In questa chiave di lettura il riconoscimento ufficiale di distretti culturali della tipologia qui soltanto abbozzata è una risorsa innovativa, capace di colmare grosse lacune dell'attuale sistema europeo preso nel suo complesso. Nel caso specifico del progetto di GoMosaico non deve sfuggire che esso si situa proprio nel cuore degli assi mediani che abbiamo individuato e che si mostra in possesso di tutte le proprietà che ne riassumono emblematicamente la natura strutturale: penetra nel vivo del territorio continentale mitteleuropeo e si affaccia sul mare Mediterraneo nel punto più strategico. Entrando in funzione, il distretto può essere dunque, ancorché in tutti i suoi limiti, un significativo e 
piccolo faro, che illumina valori e potenzialità civili indispensabili all'identità europea, altrimenti in reale pericolo di cadere nelle ombre dell'oblio, e che può offrire un possibile modello di vita culturale coinvolgente ed istruttiva. 